Running Title: PATHWAYS TO CARE AND YOUTH MENTAL HEALTH

\title{
Pathways to care of young people accessing a pilot Specialist Youth Mental Health Service in Norfolk, United Kingdom
}

Jo Hodgekins ${ }^{1,2^{*}}$, Timothy Clarke ${ }^{2}$, Hannah Cole ${ }^{2}$, Constantina Markides ${ }^{3}$, Uju Ugochukwu ${ }^{3}$, Peter Cairns ${ }^{3}$, Rebecca Lower ${ }^{2}$, David Fowler ${ }^{4}$, Jon Wilson ${ }^{1,2}$

${ }^{1}$ Department of Clinical Psychology, Norwich Medical School, University of East Anglia

${ }^{2}$ Central Norfolk Youth Service, Norfolk and Suffolk NHS Foundation Trust

${ }^{3}$ Great Yarmouth and Waveney Youth Service, Norfolk and Suffolk NHS Foundation Trust

${ }^{4}$ School of Psychology, University of Sussex

\section{*Corresponding author:}

Dr Jo Hodgekins

Clinical Lecturer in Clinical Psychology

Department of Clinical Psychology, Norwich Medical School

University of East Anglia

Norwich, NR4 7TJ

Email: j.hodgekins@uea.ac.uk Tel:+44(0)1603 591890

\section{Word Count:}

Abstract: 251 Main Text: 3445

\section{Acknowledgements}

This work was not supported by any external funding and the authors have no conflicts of interest to declare. 


\section{Abstract}

Aim: Pathways to Care $(\mathrm{PtC})$ are a means of examining and understanding routes into mental health care. It is important to examine PtC in order to identify ways in which individuals access services, as well as highlighting barriers or delays to appropriate treatment. This study aimed to examine PtC experienced by young people accessing a pilot Specialist Youth Mental Health Service (SYMHS) for those with non-psychotic, severe and complex mental health conditions in Norfolk, UK.

Methods: Data were collected on a subsample of referrals accepted in to the pilot SYMHS (N = 94) over a 12 month period. Duration and number of PtC were assessed using a semistructured interview augmented by health record examination. Measures of premorbid history, symptoms and functioning were also collected.

Results: The mean length of PtC was 3.74 years and a mean of 5.53 pathways were experienced before appropriate services were accessed. Individuals were most likely to first seek help from their General Practitioner followed by an educational provider. There were no associations between PtC and current symptoms, although individuals with a premorbid history of mental health problems experienced shorter PtC. There was a trend suggesting that individuals with long pathways had poorer functioning compared to those with shorter pathways.

Conclusions: PtC are variable in a group of young people presenting to mental health services. A majority of participants experienced 'long pathways' which may negatively impact on outcome. The results indicate the need to improve access to appropriate services by overcoming pathway barriers. Service implications are discussed.

Keywords: Adolescent, Cross-Sectional Studies, Delivery of Health Care, Mental Health, Mental Health Services 


\section{Introduction}

Pathways to Care (PtC) are a means of examining and understanding peoples' routes into mental health care (1). PtC are defined as "the sequence of contacts with individuals and organisations promoted by the distressed person's efforts, and those of his or her significant others, to seek help as well as the help that is supplied in response to these efforts" (2). These routes to care are varied and may include agencies from outside healthcare (e.g. schools, criminal justice system). Help may be sought directly by the person in distress, by people close to the individual, or by other agencies (3).

PtC have been used to explore the ways in which people with first episode psychosis access services (3). This is of particular relevance given the association between longer duration of untreated psychosis (DUP) and poorer long-term outcomes $(4,5)$. PtC information can be used to identify and target factors contributing to DUP, including delays in accessing appropriate services and other obstacles to receiving treatment.

In recent years, the rationale of early detection and intervention has been extended to encompass a broader range of mental health presentations than psychosis $(6,7)$. Indeed, $75 \%$ of all mental disorders present for the first time before the age of 24 years (8-10). Intervening early has the potential to improve outcomes (9). However, little is known about the duration or impact of treatment delays in young people presenting with non-psychotic mental health difficulties.

Despite indications that they have the greatest need, adolescents are less likely to seek help than other age groups (11). A recent systematic review of young peoples' views of mental health services highlighted a clear preference for community-based services offering a greater degree of flexibility (12). Lack of continuity of care in traditional service designs was also noted, with repeated assessments and transitions being experienced as unsettling and unhelpful. These difficulties may be exacerbated by transitions from child to adult services, which have been described as being poorly planned and executed (13). 
In line with national and international calls to improve mental health services for young people $(11,14,15)$, secondary mental health services for young people in Norfolk have recently been redesigned. This resulted in the introduction of a pilot Specialist Youth Mental Health Service (SYMHS) for young people aged 14 to 25 years with non-psychotic severe and complex mental health conditions. The aims of the service redesign were to reduce problems with transition from child to adult services, improve access to mental health services, and to provide specialist and developmentally appropriate interventions for young people at the time when most severe mental health problems emerge. The current study explores the PtC taken by a sample $(\mathrm{N}=94)$ of young people accessing the pilot service between 2012-2013. The aims of the current study were to:

1. Describe PtC for referred presenting difficulties for a sample of young people accessing the pilot SYMHS.

2. Examine relationships between PtC and baseline levels (assessments completed following acceptance in to the SYMHS) of symptoms and functioning.

\section{Methods}

Design

The study utilised a cross-sectional design, retrospectively examining PtC information for a sample of young people accessing the pilot SYMHS in Norfolk, UK. Consecutive referrals over a 12 month period (2012-2013) were interviewed between 0-3 months of being accepted into the service. Relationships between PtC, symptoms and functioning were examined.

\section{Participants}

A total of 235 young people were accepted into the pilot SYMHS between 20122013. The pilot was established to work with young people aged $14-25$ years who were experiencing severe non-psychotic mental health difficulties and poor social/occupational functioning. In addition, the service worked with all 14-25 year olds who were currently 
inpatients or at-risk of psychiatric admission. The main exclusion criteria were a diagnosis of psychosis or an eating disorder, as specialist services existed for these individuals.

Participants in the current study ( $N=94$ ) represent a convenience sample of $40 \%$ of the wider pilot Youth team cohort who consented to complete routine clinical assessments. The mean age of the sample was 18.25 years, and $71.3 \%$ were female. A premorbid history of mental health problems was present in $26.6 \%$ of the sample. These characteristics are representative of the wider pilot Youth team cohort. Further descriptive statistics about the study sample are shown in Table 1.

Participants gave permission for their data to be used anonymously as part of routinely collected service data. The study protocol and ethical considerations were reviewed and approved by the Norfolk \& Suffolk NHS Foundation Trust research governance committee (2014MH11-SE).

\section{Measures}

Pathways to Care (PtC). PtC were assessed using a semi-structured interview with the patient or significant other, augmented by examining health records $(1,16,17)$. Information relating to the patient's presenting mental health difficulty was gathered, including: source, date of onset, specific services approached, treatment offered, outcome, duration, timing and sequence of contacts. Information was also gathered about previous episodes of mental health difficulties if appropriate. This information was used to ascertain the onset of the presenting mental health problem and any premorbid mental health difficulties. Both the total length of pathways to care and the number of individual pathways accessed were of interest in describing how young people accessed the teams. Figure 1 provides further information on how information was coded from the pathways to care interview. 
Global Assessment of Functioning (GAF; 18). Functioning was rated by clinicians using the GAF. A rating between 1 (serious and persistent poor functioning) to 100 (superior functioning) was assigned to reflect an individual's current level of functioning. Scores below 50 are indicative of poor functioning.

Time Use Survey (TUS; 19). The TUS is a semi-structured interview assessing hours per week spent in a range of structured activities (work, education, voluntary work, childcare, housework, leisure and sports) over the past month. On average, a non-clinical group aged between $16-36$ years engage in 63.49 hours of structured activity per week, and activity levels below 30 hours are indicative of poor social functioning (19).

Beck Depression Inventory II (BDI-II; 20). The BDI-II is a 23-item self-report scale assessing symptoms of depression. Each item is rated on a 3-point scale of severity, with total scores ranging from 0 to 63 and higher scores indicative of more severe depression. A cut-off score of 29 is indicative of severe depression.

Social Interaction Anxiety Scales (SIAS; 21). The SIAS is a 20-item self-report scale assessing symptoms of social anxiety. Each item is rated on a 5-point (0-4) scale of severity, with total scores ranging from 0 to 80 with higher scores indicative of more severe social anxiety. A cut-off score of 43 is indicative of a clinical level of social anxiety.

\section{Analysis}

Descriptive statistics were conducted on all measures. Diagrams were used to map PtC, as per Gater et al. (1). Pearson's correlations were conducted to examine relationships between number of pathways, length of pathways in years and baseline levels of symptoms and functioning. Additionally, independent samples t-tests were conducted to examine gender differences in PtC, and differences between those with and without a premorbid history of mental health problems. PtC were also split into 'short' and 'long' subtypes based on the number of pathways experienced. Between-group comparisons on baseline measures 
of symptoms and functioning were conducted using independent t-tests to compare individuals with 'short' and 'long' pathways.

\section{Results}

\section{Descriptive data}

Participants had a mean age of onset of presenting problems of 14.51 years (median $=14.41)$, first sought help at a mean of 15.96 years of age $($ median $=15.56)$ while being accepted by appropriate services at a mean of 18.25 years (median $=17.98)$. The mean duration of help seeking delay for presenting problems was 1.36 years (median $=0.12$ ) with a mean of 2.27 (median $=1.21$ ) years duration between seeking help and accessing appropriate services and a total mean duration of 3.74 years (median $=3.05$ ) delay between onset of presenting problem and accessing services. A mean of $5.53($ median $=5)$ number of pathways between seeking help and accessing service was observed. Further descriptive statistics for PtC, baseline symptoms and social functioning are shown in Tables 1 and 2. High levels of anxiety and depression and poor functioning were observed across the sample. Table 3 shows where young people first reported seeking help. It is notable that there was often a significant delay between the onset of difficulties and seeking help.

\section{Subtypes of PtC}

Diagrams were used to map different subtypes of PtC (see Figure 2). These revealed short direct pathways (e.g. Figure 2a) in addition to complex pathways of short duration, whereby a large number of services were accessed prior to suitable referrals to mental health services (e.g. Figure 2d).Furthermore, pathways of a longer duration sometimes reflected appropriate use of stepped care approaches, where further referrals were made if the presenting difficulties did not improve (e.g. Figure $2 b$ ). Pathways which were long in both duration and in terms of the number of services accessed (e.g. Figure $2 c$ ) were also observed. 
The sample was split into two groups based on the number of services accessed during the pathway. 'Short' pathways were defined as any pathways where 3 or fewer services were accessed prior to a referral to the pilot SYMHS ( $N=32 ; 34 \%)$. This would account for the appropriate use of stepped care approaches. 'Long' pathways were defined as any pathways where 4 or more services were accessed prior to a referral to the pilot SYMHS ( $N=62 ; 66 \%)$. Descriptive statistics for baseline symptoms and functioning for individuals with 'short' and 'long' pathways are shown in Table 4. Independent samples ttests revealed no significant differences between pathway lengths and baseline levels of depression (BDI-II: short $=36.03$ vs. long $=37.79 ; t=0.65, p=.52$ ), social anxiety (SIAS: short $=41.38 \mathrm{vs}$. long $=46.65 ; t=1.38, p=.18)$, global functioning (GAF: short $=45.63 \mathrm{vs.}$ long $=$ 44.24; $t=0.49, p=.62$ ) and a trend in hours per week in structured activity (TUS: short $=$ 29.59 vs. long $=20.23 ; t=1.97, p=.06)$.

\section{Relationships between PtC, gender, symptoms and functioning}

Gender. Independent samples t-tests revealed no significant differences between males and females in terms of number of pathways (males $=5.56$ pathways vs. females $=$ 5.52 pathways; $t=0.04, p=.97$ ) or the total duration of pathways to care (males $=3.64$ years vs. females $=3.78$ years; $t=-0.18, p=.86$ ).

Premorbid history. Independent samples t-tests revealed no significant differences between individuals with and without a premorbid history of mental health problems in terms of number of pathways (5.28 vs. 5.62 pathways; $t=0.42, p=.68$ ). However, individuals with a premorbid history experienced a significantly shorter pathway duration than individuals without a premorbid history (2.14 years vs. 4.32 years; $t=2.97, p=.004$ ).

Symptoms. Pearson's correlations showed no significant associations between the total duration of the pathway and baseline levels of social anxiety $(r=-.08, p=.47)$ or depression $(r=-.004, p=.97)$. There were also no significant associations between the number of pathways accessed and baseline levels of social anxiety $(r=.12, p=.32)$ or 
depression ( $r=.09, p=.42)$. As shown in Table 4 , there were no significant differences between individuals with long and short pathways to care in terms of scores on the SIAS and BDI-II, with both groups reporting high levels of social anxiety and depression.

Functioning. Pearson's correlations showed no significant associations between the total duration of the pathway and baseline scores on the GAF $(r=.02, p=0.83)$ or TUS ( $r=-$ $.19, p=.10)$. There were also no significant associations between the number of pathways accessed and baseline scores on the GAF $(r=-.003, p=98)$ or TUS $(r=-.19, p=.09)$. In addition, GAF scores were equally low in individuals with long and short pathways. However, there was a suggestion that individuals with long pathways to care were spending fewer hours per week engaged in structured activity compared to those with short pathways, potentially indicating poorer baseline functioning in those with long pathways (see Table 4).

\section{Discussion}

\section{Summary of key findings}

$\mathrm{PtC}$ are variable in a group of young people presenting to a pilot SYMHS, both in terms of number of pathways and duration. The majority of young people experienced 'long pathways'. Although further research within a youth mental health context is required, the DUP literature suggests that early delays in an individual's journey negatively impacts on symptomatic and functional recovery in individuals with first episode psychosis (4). Therefore, it is anticipated that the delays observed within the present study may also have a potentially detrimental impact.

Additionally, it was observed that short pathway duration did not necessarily equate to a simple journey into care. Indeed, some individuals had short pathway durations, but had accessed a large number of services before their difficulties were correctly identified and a referral was made to mental health services. This indicates the need to improve and clarify access and referral routes in to appropriate services that are well known and timely. 
The study findings suggest that the majority of young people accessing secondary mental health services first seek help from their GP followed by educational settings, indicating the vital roles that these services play in overcoming pathway barriers. Additionally, the length of pathway is dependent upon both help-seeking delay and the number of services an individual is referred to prior to accessing secondary mental health care (i.e. the delay within services).

There was no association between duration or number of pathways and gender or baseline levels of anxiety or depression. However, individuals with a premorbid history of mental health problems experienced pathways of significantly shorter duration. In addition, there was a trend suggesting that those with longer pathways had poorer baseline functioning in terms of hours per week engaged in structured activity.

\section{Factors contributing to length of PtC}

Help-seeking delay (HSD). The average HSD was over 1 year and raises questions about why young people are not seeking help earlier. This may be due to a lack of awareness of mental health difficulties, poor understanding of how to access appropriate support (22, 23) and lack of age appropriate services. Young people may also experience fear, shame or embarrassment that prevent them from seeking help (24). Normalising emotional distress, targeting stigma, and promoting hope may be important in increasing help-seeking behaviour. As such, the SYMHS plans to evolve further to increase the partnership work with key partners and agencies across healthcare and universal settings. Furthermore, in order to reduce help seeking delays, it is hoped that a public mental health awareness campaign in collaboration with national anti-stigma strategies will encourage young people to access help earlier. It is also hoped that the SYMHS will incorporate a youth wellbeing service for mild-moderate mental health presentations, thereby increasing the range of young people accessing services with a shorter duration of untreated illness. 
The results suggest that young people often attempt to access help through nonhealth related services (e.g. within the education system). It is possible that such professionals may not identify mental health difficulties or are unaware of how to respond (25). This highlights the importance of raising mental health awareness in a range of settings where there are high levels of contact with young people. As such the SYMHS aims to address this by working with voluntary and third sector agencies to raise awareness of mental health difficulties in educational settings and through delivering training programmes to those in frequent contact with young people, including teachers and GPs. It is anticipated that the SYMHS will further simplify their referral routes with more emphasis being placed on self-referrals and the use of technology and social media.

Delay within services. An additional average delay of over 2 years was identified once young people had first sought help for their difficulties. This may reflect the appropriate use of stepped care models or inappropriately complex pathways where the young person is passed between services. As mental health problems emerge, young people can present with non-specific symptoms, which may be interpreted as 'normal' teenage behaviour (26). This may lead to non-acceptance of referrals or referrals to inappropriate services. Complex pathways may mean the young person has to tell their story repeatedly to different professionals, potentially leading to poor experience of services, hopelessness, or a sense of not being heard (12). Previous literature has highlighted an association between complex pathways and poor engagement (27) as well as dissatisfaction with services (28). It is vital that services for young people work together to reduce delays within services. Improving GP knowledge and raising awareness about mental health and services may also be useful in reducing delays as over $60 \%$ of young people in the current study first sought help within primary care.

\section{Factors associated with pathways to care}


Premorbid history. Individuals with a premorbid history of mental health problems experienced PtC of a significantly shorter duration than individuals without a premorbid history. It could be argued that prior experience resulted in a faster journey to care because past medical records guided referrals. In addition, previous experience of mental health difficulties may mean that individuals are quicker at identifying problems and know where to seek appropriate support. However, work needs to be done to improve individuals' access to services when mental health difficulties are emerging for the first time. As such, the SYMHS hopes to conduct research to identify groups with emerging difficulties that may find it hard to engage with services, such as young males. It is then anticipated that we will implement targeted strategies to specifically 'reach out' to 'hard to reach' groups.

Baseline functioning. There was a trend suggesting that individuals with 'long' pathways spent fewer hours per week in structured activity and therefore may demonstrate poorer baseline functioning when accessing services. Individuals with poorer functioning may be more withdrawn, have fewer social resources and therefore find it more difficult to seek help (29). However, there was no association between functioning and HSD. Moreover, the distinction between 'short' and 'long' pathways was based on number of pathways, with those with poorer baseline functioning accessing more as opposed to fewer services. Thus it may be the case that young people who present with poor functioning get 'stuck' in the system, possibly due to patterns of poor engagement (16). It is also possible that long pathways have a detrimental effect on functioning so that by the time such young people access services, functioning is reduced compared to individuals with shorter pathways. Further research is needed to understand and corroborate this relationship.

\section{Study limitations and future research}

A key strength of the current study is the use of a large sample of young people accessing secondary mental health services within a real-life clinical setting. Nevertheless, there are limitations which need to be considered. PtC were assessed retrospectively and 
were partly reliant on individuals' memory of their journey into services, which may have been influenced by their mental state. The current study also utilises cross-sectional data and only focuses on baseline symptoms and functioning. Longer-term follow-up would enable associations between PtC and outcome to be established in more detail. It is also likely that ceiling effects influenced the findings. Indeed, there were consistently high levels of symptomatology and poor functioning in the sample as a whole, making it difficult to find differences between groups. It should be noted that the current sample were accepted into the service based on their complexity (e.g. current or at-risk of inpatient admission). It would be interesting for future research to focus on PtC within wider youth mental health services. Moreover, this study reflects PtC into a pilot service and therefore accounts for pathways present prior to the introduction of a novel service design. It would be interesting to repeat this study once SYMHS is more established in order to examine whether new initiatives can have a positive effect in reducing the number and length of $\mathrm{PtC}$, and whether this is linked with improvements in symptomatic and functional outcomes.

\section{Conclusions}

This study highlights the complex PtC experienced by young people with mental health difficulties. The long-term effects of lengthy and complex PtC are unknown but these are unlikely to be ideal. There is a need for new service models to consider PtC and to attempt to make these as short as possible, both by improving access to young people in distress and by streamlining processes once young people have sought help. The impact of such initiatives on the experiences and outcomes of young people will be an important focus of future research. 


\section{References}

1. Gater R, de Almeida e Sousa B, Barrientos G, Caraveo J, Chandrashekar CR, Dhadphale M, et al. The pathways to psychiatric care: a cross-cultural study. Psychol Med 1991; 21: 761-74.

2. Rogler LH, Cortes DE. Help-seeking pathways: a unifying concept in mental health care. Am J Psychiatry 1993; 150: 554-61.

3. Singh SP, Grange T. Measuring pathways to care in first-episode psychosis: a systematic review. Schizophr Res 2006; 81: 75-82.

4. Marshall M, Lewis S, Lockwood A, Drake R, Jones P, Croudace T. Association between duration of untreated psychosis and outcome in cohorts of first-episode patients: a systematic review. Arch Gen Psychiatry 2005; 62: 975-83.

5. Crumlish N, Whitty P, Clarke M, Browne S, Kamali M, Gervin M, et al. Beyond the critical period: longitudinal study of 8-year outcome in first-episode non-affective psychosis. Br J Psychiatry 2009; 194: 18-24.

6. Fusar-Poli P, Yung AR, McGorry P, van Os J. Lessons learned from the psychosis highrisk state: towards a general staging model of prodromal intervention. Psychol Med 2014; 44: 17-24.

7. Fowler D, Hodgekins J, Arena K, Turner R, Lower R, Wheeler K, et al. Early detection and psychosocial intervention for young people who are at risk of developing long term socially disabling severe mental illness: should we give equal priority to functional recovery and complex emotional dysfunction as to psychotic symptoms? Clin Neuropsychiatry 2010; 7: 63-71.

8. Kim-Cohen J, Caspi A, Moffitt TE, Harrington H, Milne BJ, Poulton R. Prior juvenile diagnoses in adults with mental disorder: developmental follow-back of a prospectivelongitudinal cohort. Arch Gen Psychiatry 2003; 60: 709-17. 
9. Kessler RC, Berglund P, Demler O, Jin R, Merikangas KR, Walters EE. Lifetime prevalence and age-of-onset distributions of DSM-IV disorders in the National Comorbidity Survey Replication. Arch Gen Psychiatry 2005; 62: 593-602.

10. McGorry PD, Purcell R, Hickie IB, Yung AR, Pantelis C, Jackson HJ. Clinical staging: a heuristic model for psychiatry and youth mental health. MJA 2007; 187: S40-2.

11. Young Minds. A Call to Action: Comissioning mental health services for $16-25$ year olds. London: Young Minds; 2006.

12. Plaistow J, Masson K, Koch D, Wilson J, Stark RM, Jones PB, et al. Young people's views of UK mental health services. Early Interv Psychiatry 2014; 8: 12-23.

13. Singh SP, Paul M, Ford T, Kramer T, Weaver T, McLaren S, et al. Process, outcome and experience of transition from child to adult mental healthcare: multiperspective study. Br J Psychiatry 2010; 197: 305-12.

14. McGorry PD. The specialist youth mental health model: strengthening the weakest link in the public mental health system. MJA 2007; 187: S53-6.

15. Department of Health. Closing the Gap: Priorities for essential change in mental health. London: DoH; 2014.

16. Birchwood M, Connor C, Lester H, Patterson P, Freemantle N, Marshall M, et al. Reducing duration of untreated psychosis: care pathways to early intervention in psychosis services. Br J Psychiatry 2013; 203: 58-64.

17. Birchwood $M$, Lester $H$, McCarthy L, Jones $P$, Fowler $D$, Amos $T$, et al. The UK national evaluation of the development and impact of Early Intervention Services (the National EDEN studies): study rationale, design and baseline characteristics. Early Interv Psychiatry 2014; 8: 59-67.

18. American Psychiatric Association. Global Assessment of Functioning. DSM-IV-TR: American Psychiatric Association; 2000. p. 32. 
19. Hodgekins J, French P, Birchwood M, Mugford M, Christopher R, Marshall M, et al. Comparing time use as a measure of social functioning in individuals at different stages of psychosis and in a non-clinical comparison group. Schizophr Res 2015; 161: 188-93.

20. Beck AT, Steer RA, Brown GK. BDI-II Manual. San Antonio, TX: The Psychological Corporation; 1996.

21. Mattick RP, Clarke JC. Development and validation of measures of social phobia scrutiny fear and social interaction anxiety. 1989.

22. Wright A, McGorry PD, Harris MG, Jorm AF, Pennell K. Development and evaluation of a youth mental health community awareness campaign: The Compass Strategy. BMC Public Health 2006; 6: 215.

23. Gulliver A, Griffiths KM, Christensen H. Perceived barriers and facilitators to mental health help-seeking in young people: a systematic review. BMC Psychiatry 2010; 10: 113.

24. Young Minds. A review of the evidence. London: Young Minds; 2010.

25. Kelly $\mathrm{CM}$, Jorm $\mathrm{AF}$, Wright $\mathrm{A}$. Improving mental health literacy as a strategy to facilitate early intervention for mental disorders. MJA 2007; 187: S26-30.

26. Addington J, Van Mastrigt S, Hutchinson J, Addington D. Pathways to care: help seeking behaviour in first episode psychosis. Acta Psychiatr Scand 2002; 106: 358-64.

27. Compton MT. Barriers to initial outpatient treatment engagement following first hospitalization for a first episode of nonaffective psychosis: a descriptive case series. J Psychiatr Pract 2005; 11: 62-9.

28. Bhugra D, Harding C, Lippett R. Pathways into care and satisfaction with primary care for black patients in South London. J Ment Health 2004; 13: 171-83.

29. Pisani AR, Schmeelk-Cone K, Gunzler D, Petrova M, Goldston DB, Tu X, et al. Associations between suicidal high school students' help-seeking and their attitudes and perceptions of social environment. J Youth Adolesc 2012; 41: 1312-24. 
Table 1. Descriptive Statistics of Sample

\begin{tabular}{lccc}
\hline & $\mathrm{N}$ & Mean (SD) & Median \\
\hline Age first experienced mental health problems (years) & 94 & $12.71(3.51)$ & 13.56 \\
Age of onset of presenting problem (years) & 94 & $14.51(3.02)$ & 14.41 \\
Age first sought help for presenting problem (years) & 89 & $15.96(2.72)$ & 15.56 \\
Age accepted by service (years) & 94 & $18.25(2.86)$ & 17.98 \\
Beck Depression Inventory-II score (0-63) & 77 & $37.13(11.43)$ & 37.00 \\
Social Interaction Anxiety Scale score & 78 & $44.69(15.27)$ & 46.00 \\
Global Assessment of Functioning score (0-100) & 94 & $44.71(12.83)$ & 45.50 \\
Time Use Survey score (hours per week in structured activity) & 80 & $23.62(18.82)$ & 18.67 \\
\hline
\end{tabular}

Table 2. Pathways to Care Descriptive Statistics

\begin{tabular}{|c|c|c|c|c|}
\hline & $\mathrm{N}$ & Mean (SD) & Min-Max & Median \\
\hline Premorbid history duration (years) & 25 & $6.77(3.64)$ & $1.37-18.08$ & 6.13 \\
\hline $\begin{array}{l}\text { Duration of help seeking delay for } \\
\text { presenting problem (A) }\end{array}$ & 89 & $1.36(2.41)$ & $0.00-10.59$ & .12 \\
\hline $\begin{array}{l}\text { Duration between seeking help and } \\
\text { accessing service (B) }\end{array}$ & 89 & $2.27(2.57)$ & $0.05-11.84$ & 1.21 \\
\hline $\begin{array}{l}\text { Number of pathways between seeking help } \\
\text { and accessing service }\end{array}$ & 94 & $5.53(3.50)$ & $1.00-20.00$ & 5.00 \\
\hline $\begin{array}{l}\text { Total duration of delay (years) between } \\
\text { onset of presenting problem and accessing } \\
\text { service (C) }\end{array}$ & 94 & $3.74(3.28)$ & $0.06-12.84$ & 3.05 \\
\hline
\end{tabular}


Table 3. Frequency of first pathway

\begin{tabular}{lc}
\hline & $\mathrm{N}(\%)$ \\
\hline General Practitioner/Primary Care & $59(62.8)$ \\
Education & $14(14.9)$ \\
A\&E/Crisis & $8(8.5)$ \\
Social Care & $6(6.4)$ \\
Other (e.g. Criminal Justice System, private & $7(7.4)$ \\
care providers, physical health services) &
\end{tabular}

Table 4. Between-group differences

Mean (SD)

Short pathway Long pathway Independent t-test

(3 or less) (4 or more)

\begin{tabular}{lccc}
\hline Beck Depression Inventory-II score (0-63) & $36.03(10.59)$ & $37.79(11.97)$ & $t=-0.65, p=.52$ \\
Social Interaction Anxiety Scale score & $41.38(17.97)$ & $46.65(13.22)$ & $t=-1.38, p=.18$ \\
Global Assessment of Functioning score (0-100) & $45.63(9.92)$ & $44.24(14.15)$ & $t=0.49, p=.62$ \\
Time Use Survey score (hours per week in & $29.59(22.91)$ & $20.23(15.28)$ & $t=1.97, p=.06$ \\
structured activity) & & &
\end{tabular}


Figure 1. Description of information collection from Pathways to Care interview

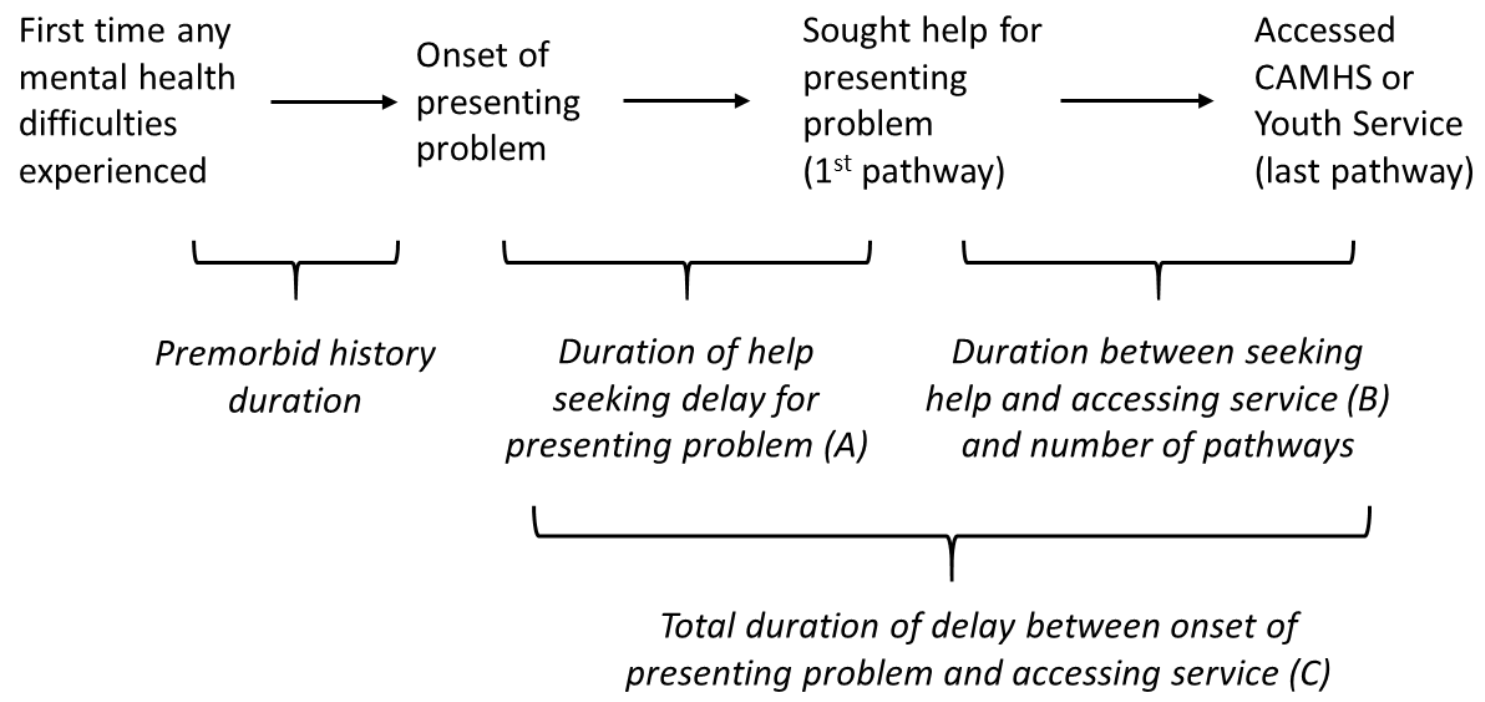


Figure 2. Diagrams of different types of pathways to care

(a) Example of short "direct" pathway (0.49 years)

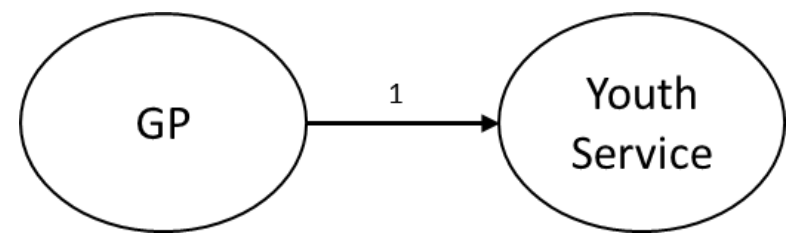

(b) Example of short "stepped-care" pathway (3.53 years)

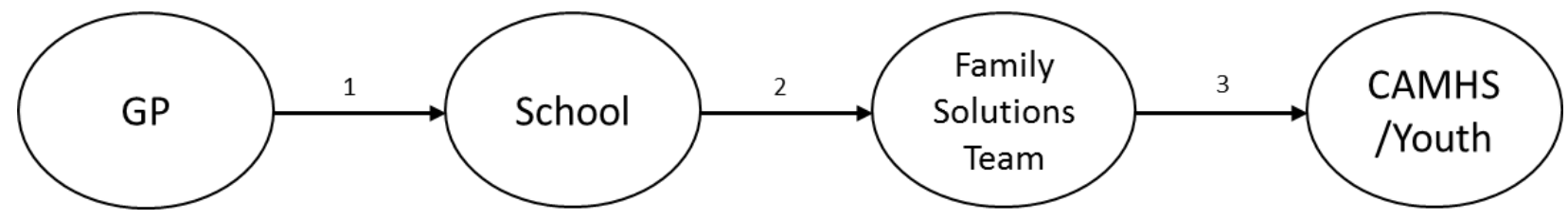


(c) Example of complex pathway of long duration (4.80 years)

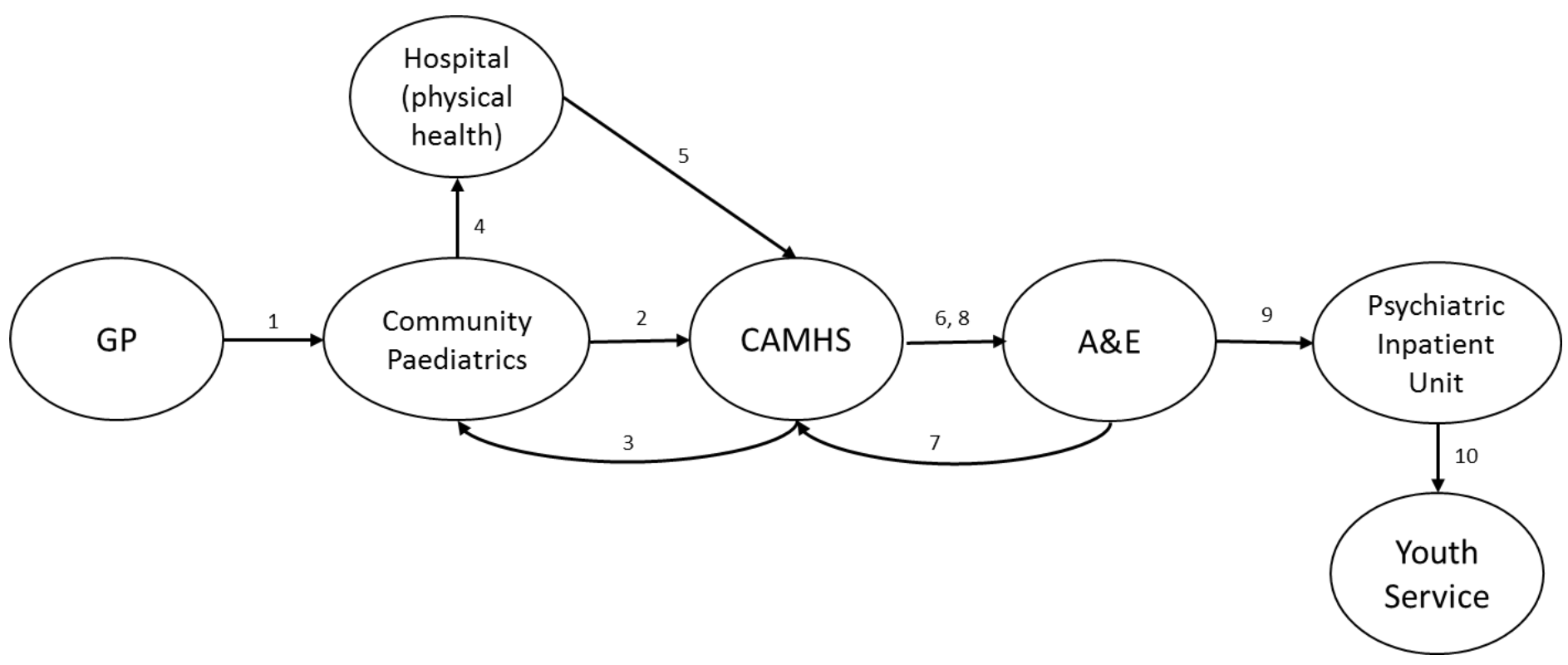


(d) Example of complex pathway of short duration (1.07 years)

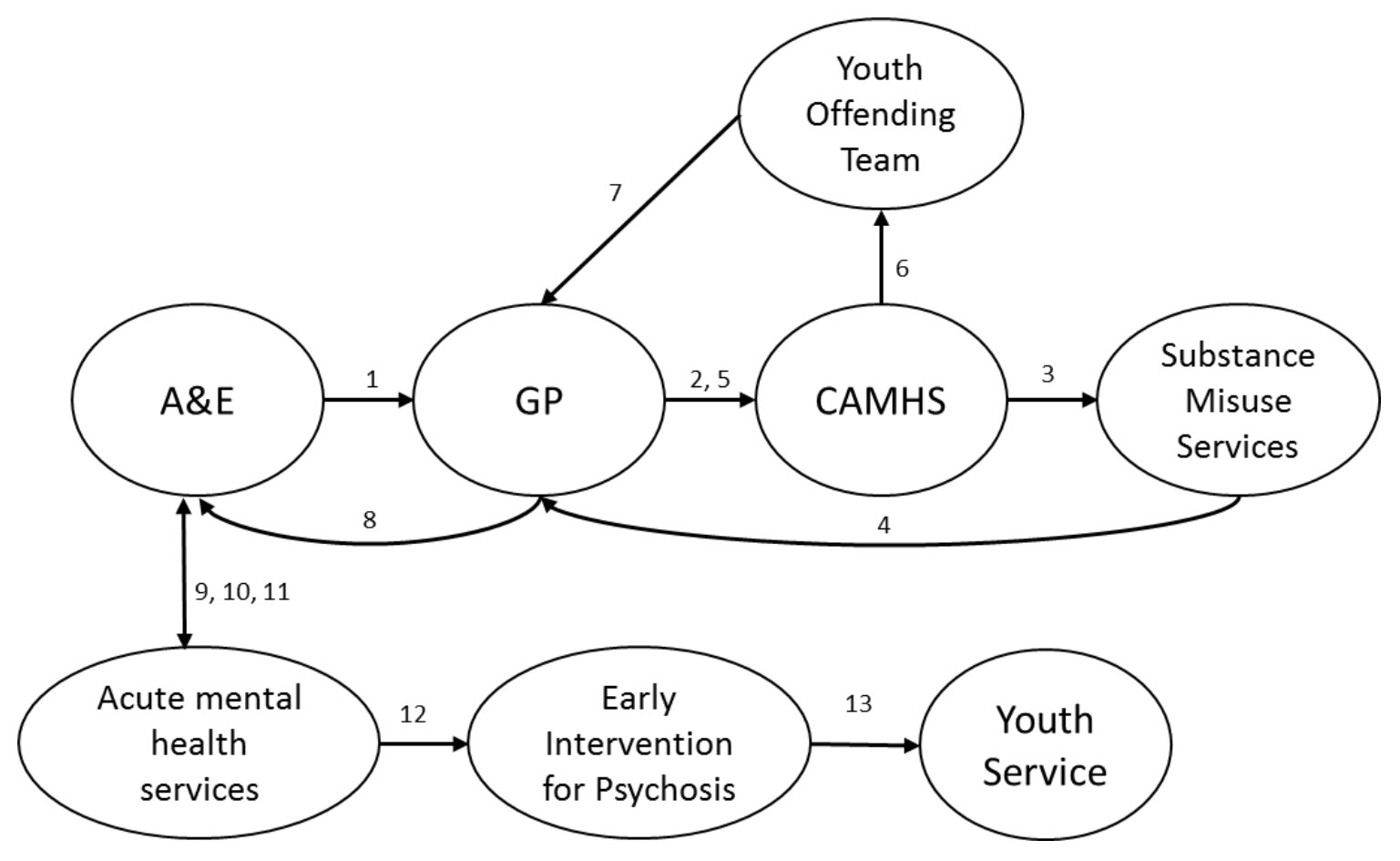

Note. Order of pathways is indicated by the pathway number on each of the arrows 